\title{
Induction of labour - a misused blessing: Prospective study of factors influencing the success of induction and comparison of fetomaternal outcomes with spontaneous labour
}

\author{
V Vinod, MB BS, DGO, DNB (OBG); R G Bhat, MB BS, MD (OBG); P V Bhat, MB BS, DGO, MD (OBG); \\ N Zahoor, MB BS, MD (OBG) S Bhat, MB BS, MD (OBG)
}

Department of Obstetrics and Gynaecology, Manipal Academy of Higher Education, Kartnataka, India

Corresponding author: V Vinod (vinuthakvinod@gmail.com)

\begin{abstract}
Background. Although most pregnant women experience spontaneous labour at term, induction of labour is indicated whenever continuation of pregnancy is detrimental to either the fetus or the mother.

Objectives. To study the factors associated with the successful induction of labour and to compare the maternal and fetal outcomes between induced and spontaneous labour.

Methods. We conducted a prospective observational and comparative study from September 2015 to December 2016 at Dr TMA Pai Hospital a secondary level hospital at Manipal Academy of Higher Education (MAHE). Women with a singleton pregnancy, live fetus, vertex presentation and gestational age $(\mathrm{GA})>36$ weeks were included in the study, and those with antepartum haemorrhage, scarred uterus, anomalous fetus and intrauterine fetal demise were excluded.

Results. Out of 1575 deliveries during the study period, 550 were induced (34\%). A total of 300 inductions fulfilled the inclusion criteria and formed the study group. Multiparity, body mass index (BMI) $<25$ and GA $>38$ weeks were factors associated with successful induction. Among the components of the Bishop score, dilatation was a better predictor of vaginal delivery $(p<0.001)$ and post-dated pregnancy was the most common indication (33.6\%). The rates of caesarean section (CS) delivery (33\% v. 12\%) and neonatal intensive care unit (NICU) admissions (4\% v. $1 \%)$ were more in the induced group compared with the spontaneous group.

Conclusion. Multiparity, BMI $<25$ and advancing GA are predictors of successful induction. Induction is safe but carries a high risk of CS delivery and NICU admissions.

Keywords. labour induction; successful induction; caesarean section.

S Afr J Obstet Gynaecol 2020;26(3):84-88. https://doi/org/10.7196/SAJOG.2020.v26i3.1593
\end{abstract}

Induction of labour (IOL) is defined as the initiation of contractions in a pregnant woman who is not in labour to have a vaginal birth within $24-48$ hours. ${ }^{[1]}$ Although most pregnant women experience spontaneous labour at term, IOL is indicated whenever continuation of the pregnancy is detrimental either to the fetus or the mother. Public and private sector hospitals provide maternity care in India. The management, facilities and resources are varied among these. The rising caesarean section (CS) rates in developing countries are alarming. IOL is known to be associated with increased CS deliveries. ${ }^{[2,3]}$ The IOL rate is increasing due to a rise in medically and obstetrically indicated inductions, as well as elective inductions. Elective induction is the IOL in the absence of acceptable fetal or maternal indications. ${ }^{[1]}$ Increased public awareness regarding management of pregnancy and childbirth, obstetrician's prior experience with high-risk pregnancies, increased litigation and waning trust in the healthcare provider have forced obstetricians to play it safe by inducing pregnancy for borderline indications.

IOL is also associated with increased uterotonic use, perineal lacerations, hysterectomy, intensive care unit (ICU) and neonatal intensive care unit (NICU) admission, longer hospital stays, greater anaesthesia/analgesia requirements during labour and lower Apgar scores..$^{[4,5]}$

\section{Methods}

This study was conducted at Dr TMA Pai Hospital, Udupi - a secondary level hospital at the Manipal Academy of Higher Education (MAHE). This was a prospective observational and comparative study conducted from September 2015 to December 2016. Women with a singleton pregnancy, live fetus, vertex presentation and gestational age (GA) $>36$ weeks were included in the study and those with antepartum haemorrhage, scarred uterus, anomalous fetus and intrauterine fetal demise were excluded.

Ethical clearance was obtained from the institutional ethics committee (ref. no. MUEC/13/2015-2016). To estimate a $25 \%$ incidence of induced labour at $95 \%$ confidence with a relative precision of $20 \%$, a minimum of 288 antenatal women needed to be included in the study. A total of 300 women consented to participate in the study; they were induced, and their outcomes compared with women who had consecutive spontaneous labour. Method and number of inductions were documented, and the progress of labour 
was plotted on the partograph. Mode of delivery, indications for operative delivery, morbidity in mother and fetus/neonate, if any, were noted.

Bishop score assessment was done post their consent. Patients were induced with intracervical prostaglandin E2 (PGE2) gel $(0.5 \mathrm{mg})$ or vaginal misoprostol $(25 \mu \mathrm{g})$. Pre- and post-induction cardiotocography was done.

The patients were reassessed after 6 hours and depending on the findings, they were either reinduced with PGE2 gel (maximum 3 doses) or misoprostol (maximum 5 doses), or labour was augmented with oxytocin or amniotomy.

Following induction, if the cervical dilatation was $>3 \mathrm{~cm}$ (active phase), it was considered as the patient having responded to induction or had a successful induction. Failed induction was considered as failure to enter the active phase of labour.

\section{Data analysis}

Data were entered and analysed using SPSS 16 software (SPSS Inc., USA). The percentage was used to summarise categorical data, and mean and standard deviation (SD) or median and interquartile range (IQR) was used to summarise continuous data depending on the skewness. A $\chi^{2}$ test was used to test for associations. Odds ratio (OR) or relative risk (RR) with 95\% confidence interval (CI) was provided. Logistic regression was used to identify factors influencing the success of induction of labour.

\section{Results}

There were 1575 deliveries and 550 were induced (induction rate of $34 \%$ ) at the hospital during the study period. A total of 300 induced women were included in the present study.

Post-dated ( $>40$ weeks) pregnancy (33.6\%; $n=101)$ was the most common indication for IOL, followed by indications such as intrauterine growth restriction (8.6\%; $n=26)$, oligohydramnios $\quad(21 \%$; $n=63)$, pre-labour rupture of membranes $(14.3 \% ; n=43)$ and medical disorders in pregnancy (18.2\%; $n=55)$, and some women $(9 \% ; n=27)$ were induced electively (Fig. 1). The indications were divided to absolute and relative for analysis. Absolute indications included medical disorders, growth-restricted fetuses, severe oligohydramnios and pre-labour rupture of membranes $(42.7 \% ; n=128)$, and relative indications included past-dated pregnancy,

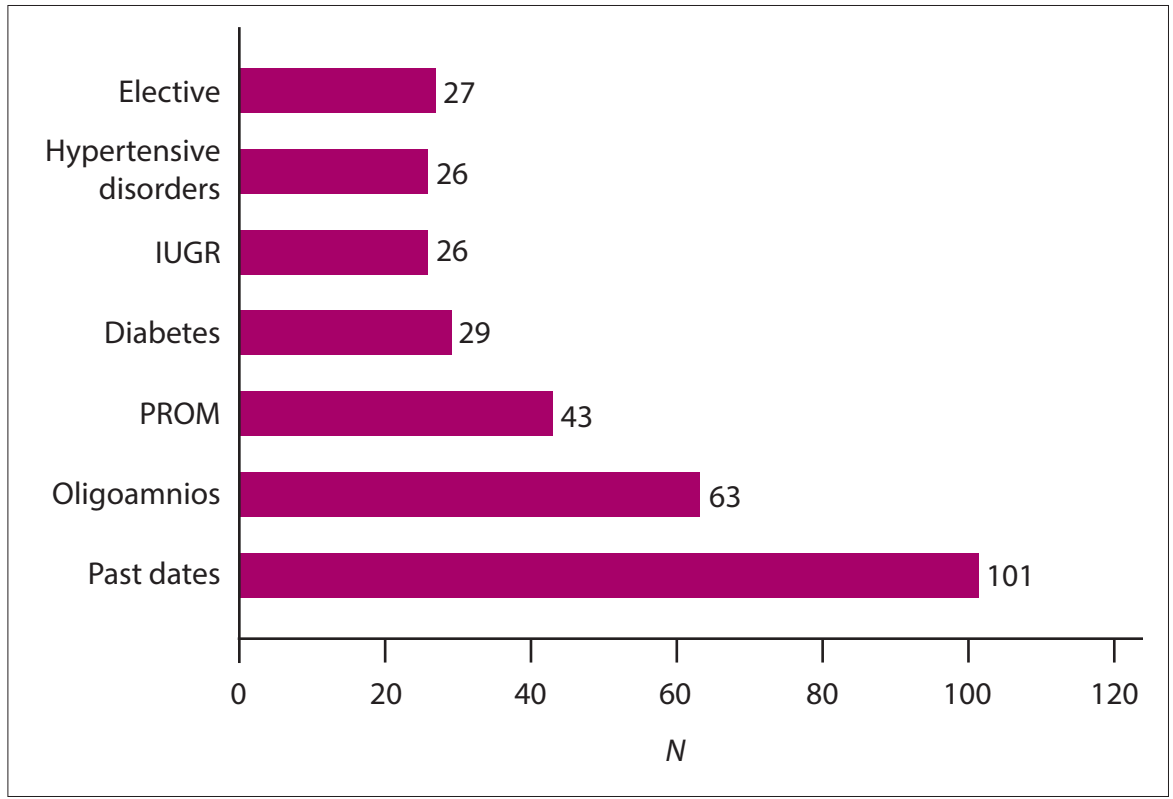

Fig. 1. Indications for induction of labour. $N=315$, as a few patients had multiple indications. (IUGR = intrauterine growth restriction; $P R O M=$ premature rupture of membranes.)

borderline oligohydramnios $\quad(48.3 \%$; $n=145)$ and elective induction $(9 \% ; n=27)$. The mean (SD) age of women who were induced was 27 (3) years. The majority of the women were induced with PGE2 (85\%; $n=255$ ).

\section{Response rate/ successful induction}

The majority of the women $(74.7 \%$; $n=224 / 300$ ) responded to induction and entered the active phase of labour. Two-thirds of the women $(66.3 \% ; n=199 / 300)$ delivered vaginally and $33.7 \% \quad(n=101)$ of women underwent CS.

The response rate was $94 \%(n=78 / 83)$ in multigravida and 67\% $(n=146 / 217)$ in nullipara women $(p<0.0001)$. Women with BMI $<25 \mathrm{~kg} / \mathrm{m}^{2}$ responded better (82\%; $n=142 / 172) \quad$ compared with women with BMI $>25.1 \mathrm{~kg} / \mathrm{m}^{2}$ (64\% $(n=82 / 128) ; p=0.001)$. The response rate was better when the GA was $>38$ weeks compared with GA $<38$ weeks $(79 \% \quad$ v. $62 \% ; \quad p=0.009) \quad$ (Table 1). Women with a higher Bishop score (>6) showed a response rate of $83.8 \%$ $(n=26 / 31)$, and $73.6 \% \quad(n=198 / 269)$ of them responded when it was $<5$. When the individual components of the Bishop score were analysed, $89 \% \quad(n=65 / 73)$ of the women with cervical dilatation $>2 \mathrm{~cm}$ responded compared with $70 \%$ $(n=159 / 227)$ of women with dilatation $<1 \mathrm{~cm}(p<0.001)$. Women whose cervix was mid-positioned, soft and had a length $<2 \mathrm{~cm}$ were more likely to respond to induction. Women with the station of fetal vertex below -2 were 1.13 times more likely to respond to induction compared with those with a higher station.

\section{Vaginal delivery}

The vaginal delivery rate in induced women was $66 \%(n=199 / 300)$. The median (IQR) induction to the delivery interval was 12.3 (8 - 24) hours. The vaginal delivery rate in multiparous women was $81 \%(n=67 / 83)$ and $59 \%(n=128 / 217)$ in nulliparous women. Women with BMI <25, advanced GA $>38$ weeks and Bishop score $>6$ had a good successful vaginal delivery rate (Table 2 ). We also analysed whether the indication for induction had any influence on the outcome of IOL. Women who were induced for postdated pregnancies had the highest vaginal delivery rate $(75.2 \% ; n=76 / 101)$. Furthermore, $48 \%(n=13 / 27)$ of women who had elective induction had vaginal deliveries.

A multivariable logistic regression analysis was conducted to determine the influence of factors such as high-risk pregnancy, period of gestation, maternal height and parity on CS rate in induced women. It was observed that only parity (nullipara v. multipara) had a significant influence $(p<0.001)$ with an adjusted OR $(95 \%$ CI) of $4.2(2.1-8.3)$. Obstetric and neonatal outcome of induced women were compared with those who had a spontaneous onset of labour. The demographic details are provided in Table 3. The number of primigravidae was high in the induced group. 
RESEARCH

Table 1. Factors influencing the success of induction $(N=300)$

\begin{tabular}{|c|c|c|c|c|}
\hline Factor & $n$ & Responded $n(\%)$ & RR $(95 \% \mathrm{CI})$ & $p$-value \\
\hline \multicolumn{5}{|l|}{ Age, years } \\
\hline$<34$ & 285 & $213(74.7)$ & \multirow[t]{2}{*}{$1.02(0.7-1.39)$} & \multirow[t]{2}{*}{0.922} \\
\hline$>35$ & 15 & $11(73)$ & & \\
\hline \multicolumn{5}{|l|}{ Parity } \\
\hline Multigravida & 83 & $78(94)$ & \multirow[t]{2}{*}{$5.3(2.2-12.6)$} & \multirow[t]{2}{*}{$<0.001$} \\
\hline Primigravida & 217 & $146(67)$ & & \\
\hline \multicolumn{5}{|l|}{ GA, weeks } \\
\hline$>38.1$ & 218 & $171(79.9)$ & \multirow[t]{2}{*}{$2(1.1-3.6)$} & \multirow[t]{2}{*}{0.009} \\
\hline$<38$ & 82 & $62(53)$ & & \\
\hline \multicolumn{5}{|l|}{$\mathrm{BMI}, \mathrm{kg} / \mathrm{m}^{2}$} \\
\hline$<25$ & 172 & $142(82)$ & \multirow[t]{2}{*}{$1.3(1.1-1.5)$} & \multirow[t]{2}{*}{$<0.001$} \\
\hline$>25.1$ & 128 & $82(64)$ & & \\
\hline \multicolumn{5}{|l|}{ Bishop score } \\
\hline$>6$ & 31 & $26(83.8)$ & \multirow{2}{*}{$1.86(0.7-6.45)$} & \multirow[t]{2}{*}{0.277} \\
\hline$<5$ & 269 & $198(73.6)$ & & \\
\hline \multicolumn{5}{|l|}{ Position } \\
\hline Anterior & 12 & $8(66.6)$ & \multirow{3}{*}{$\begin{array}{l}0.8(0.2-2.8) \\
1.7(0.9-3)\end{array}$} & \multirow{3}{*}{$\begin{array}{l}0.718 \\
0.068\end{array}$} \\
\hline Mid & 126 & $101(80.2)$ & & \\
\hline Posterior & 162 & $111(70.9)$ & & \\
\hline \multicolumn{5}{|l|}{ Consistency } \\
\hline Soft & 214 & $61(75.2)$ & \multirow{3}{*}{$\begin{array}{l}2.1(0.3-13) \\
1.9(0.2-12)\end{array}$} & \multirow{3}{*}{$\begin{array}{l}0.414 \\
0.496\end{array}$} \\
\hline Medium & 81 & $60(74.1)$ & & \\
\hline Firm & 5 & $3(60)$ & & \\
\hline \multicolumn{5}{|l|}{ Length, $\mathrm{cm}$} \\
\hline$<2$ & 113 & $91(80)$ & \multirow[t]{2}{*}{$1.12(0.97-1.27)$} & \multirow[t]{2}{*}{0.157} \\
\hline$>3$ & 187 & $133(71)$ & & \\
\hline \multicolumn{5}{|l|}{ Dilatation } \\
\hline$<2$ & 227 & $159(70)$ & \multirow[t]{2}{*}{$2.7(1.3-5.4)$} & \multirow[t]{2}{*}{$<0.001$} \\
\hline$>3$ & 73 & $65(89)$ & & \\
\hline \multicolumn{5}{|l|}{ Station } \\
\hline-3 & 227 & $164(72.2)$ & \multirow[t]{2}{*}{$1.13(0.9-1.3)$} & \multirow[t]{2}{*}{0.058} \\
\hline$-2-1$ & 73 & $60(82)$ & & \\
\hline
\end{tabular}

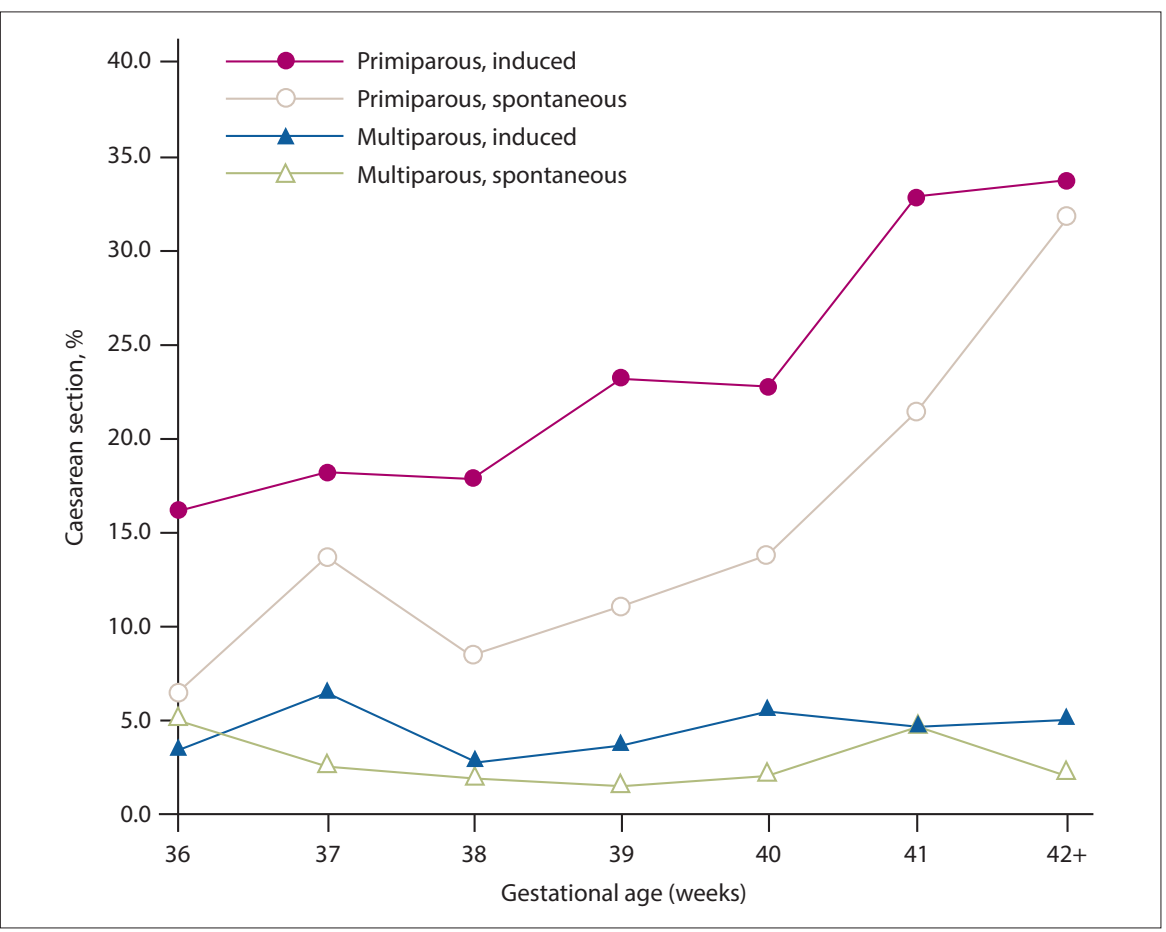

Fig. 2. Caesarean rates in induced and spontaneous groups.
The median duration of the latent, active and second stages of labour were comparable in both groups. The vaginal delivery rate in the spontaneous group was $87.3 \% \quad(n=262 / 300)$ compared with $66.3 \%(n=199 / 300)$ in the induced group $(p<0.001)$. The rate of CS delivery in the induced group was $33 \% \quad(n=101 / 300)$ compared with $12 \%(n=38 / 300)$ in the spontaneous group (Table 4). The CS rate was higher in nulliparous than multiparous women in both groups (Fig. 2).

The most common indication for CS in the induced group was failed induction $(46.5 \% ; n=47 / 101)$. Fetal distress was observed more often in the induced group $(11.6 \% ; n=35 / 300)$ than in the spontaneous group (4\%; $n=13 / 300)$. Meconium staining of liquor was seen in $2.3 \%(n=7 / 300)$ of women in the induced group compared with $4 \%(n=12 / 300)$ in the spontaneous group. There was an arrest of dilatation in $5 \% \quad(n=15 / 300)$ and $3 \% \quad(n=10 / 300)$ of the induced and spontaneous groups, respectively. Instrumental delivery was low in the induced group. The mean birth weight and Apgar scores were similar in both groups (Table 4). The incidence of atonic postpartum haemorrhage was marginally higher in the induced group than in the spontaneous group (19v. 12).

Less than a tenth of babies $(4 \% ; n=12)$ born from women in the induced group had morbidities such as sepsis $(n=7)$, respiratory distress $(n=2)$, meconium aspiration $(n=2)$ and hypoglycaemia $(n=1)$ compared with $1 \%(n=3)$ in the spontaneous group who had hypoglycaemia $(n=1)$, respiratory distress $(n=1)$ and meconium aspiration $(n=1) \quad(p=0.018)$. Women induced for pre-labour rupture of membranes had higher neonatal morbidity $(50 \% ; n=6 / 12)$.

The mean (SD) duration of stay in hospital was 6 (2) days in the induced group compared with 4 (1.7) days in the spontaneous group.

\section{Discussion}

A World Health Organization (WHO) global survey on maternal and neonatal health showed an induction rate of $12 \%$ in Asian countries. ${ }^{[5]}$ The induction rate was higher in our study compared with studies by Zenzmaier et al. ${ }^{[6]}$ and Abisowa et al. ${ }^{[3]}$ who documented an induction rate of $19.7 \%$ and $16 \%$, respectively. The higher rate of induction may be attributed to the number of medically indicated inductions $(18.2 \%$; $n=54 / 300)$, our policy to 
Table 2. Factors influencing vaginal delivery

\begin{tabular}{lllll}
\hline Factor & $\boldsymbol{n}$ & $\begin{array}{l}\text { Vaginal } \\
\text { delivery, } \boldsymbol{n}(\%)\end{array}$ & $\mathbf{R R}(\mathbf{9 5} \% \mathbf{C I})$ & $\boldsymbol{p}$-value \\
\hline Age, years & & & & \\
$\quad>35$ & 15 & $8(53)$ & $1.2(0.7-2.0)$ & 0.29 \\
$\quad<34$ & 285 & $191(66.7)$ & & \\
$\begin{array}{l}\text { Parity } \\
\quad \text { Primigravida }\end{array}$ & 217 & $128(59)$ & $2.3(1.6-4.9)$ & $<0.001$ \\
$\quad$ Multigravida & 83 & $67(81)$ & & \\
BMI, kg/m & & & & \\
$\quad>25.1$ & 128 & $76(59)$ & $1.4(1.0-1.9)$ & 0.04 \\
$\quad<25$ & 172 & $123(71.5)$ & & \\
GA, weeks & & & & \\
$\quad<38$ & 85 & $45(53)$ & $1.3(1.0-1.5)$ & 0.002 \\
$\quad>38$ & 215 & $154(72)$ & & \\
Bishop score & & & & \\
$\quad<5$ & 269 & $175(65)$ & $1.5(0.8-3.0)$ & 0.228 \\
$\quad \geq 6$ & 31 & $24(77)$ & &
\end{tabular}

$\mathrm{RR}=$ relative risk $\mathrm{CI}=$ confidence interval; $\mathrm{BMI}=$ body mass index; $\mathrm{GA}=$ gestational age.

Table 3. Demographic data comparison between spontaneous and induced group

\begin{tabular}{lll}
\hline Variable & Induction, $\boldsymbol{n}(\%)^{*}$ & Spontaneous, $\boldsymbol{n}(\%)^{*}$ \\
\hline Age (weeks), mean (SD) & $27(3)$ & $27(3)$ \\
Parity & & $189(63)$ \\
$\quad$ Primigravida & $217(72)$ & $111(37)$ \\
$\quad$ Multigravida & $83(28)$ & $38.4(0.6)$ \\
GA (weeks), mean (SD) & $39(0.4)$ & $23.8(4.29)$ \\
BMI, mean (SD) & $24.2(4.6)$ & \\
SD = standard deviation; GA = gestational age; BMI = body mass index. \\
*Unless otherwise specified.
\end{tabular}

Table 4. Comparison of outcome between spontaneous and induced labor groups

\begin{tabular}{|c|c|c|c|}
\hline Variable & $\begin{array}{l}\text { Induction, } \\
n(\%)^{\star}\end{array}$ & $\begin{array}{l}\text { Spontaneous, } \\
n(\%)^{*}\end{array}$ & $p$-value \\
\hline $\begin{array}{l}\text { Latent phase }(\mathrm{h}) \text {, } \\
\text { median (IQR) }\end{array}$ & $4.45(3-8)$ & $4(3-8)$ & 0.035 \\
\hline $\begin{array}{l}\text { Active phase }(\mathrm{h}) \text {, } \\
\text { median (IQR) }\end{array}$ & $2.1(1.3-4.0)$ & $3(2.0-4.3)$ & 0.606 \\
\hline $\begin{array}{l}\text { Second stage }(\mathrm{min}) \text {, } \\
\text { median (IQR) }\end{array}$ & $15(0.1-0.3)$ & $15(0.12-0.25)$ & 0.578 \\
\hline \multicolumn{4}{|l|}{ Mode of delivery } \\
\hline Vaginal & $199(66.3)$ & $262(87.3)$ & $<0.0001$ \\
\hline LSCS & $101(33.7)$ & $38(12.7)$ & \\
\hline Instrumental & $14 / 199(7)$ & $22 / 262(8)$ & 0.597 \\
\hline Analgesia & $89(29)$ & $73(24)$ & 0.141 \\
\hline $\begin{array}{l}\text { Birthweight (g), } \\
\text { mean (SD) }\end{array}$ & $3041(395)$ & $3041(406)$ & - \\
\hline \multicolumn{4}{|l|}{ Apgar (>7) } \\
\hline $1 \mathrm{~min}$ & $298(99.3)$ & $299(99.6)$ & - \\
\hline $5 \mathrm{~min}$ & $300(100)$ & $300(100)$ & \\
\hline
\end{tabular}

induce at 40 weeks of GA, inductions for minor indications such as borderline reduced amniotic fluid index and elective inductions.

The most common indication for induction in our study was post-dated pregnancy $(33 \% ; n=101 / 300)$, followed by oligohydramnios $(21 \% ; n=63 / 300)$. We induce at 40 weeks of GA as studies conducted in India ${ }^{[7,8]}$ have shown that fetuses are more likely to mature early and the incidence of meconium staining of amniotic fluid increases when gestation crosses 280 days. We perform routine ultrasound for the growth of the fetus at $28-32$ weeks and weekly amniotic fluid assessment for all women after 36 weeks of gestation. This could lead to overdiagnosis of growth restriction and oligohydramnios and, in turn, increase inductions for borderline indications.

The factors that influence the outcome of induction were studied and it was observed that women $<34$ years of age, multiparous, BMI $<25 \mathrm{~kg} / \mathrm{m}^{2}$ and GA $>38$ weeks had successful inductions. Pevzner et al..$^{[9]}$ has also shown that all the above factors need to be considered for predicting successful induction.

The collagen of the cervix in multiparous women is damaged permanently by pregnancy, so ripening is more readily accomplished in subsequent pregnancies. Khan et al. ${ }^{[10]}$ found that nulliparity had the strongest association with failed induction. Likewise, Dammer et al. ${ }^{[1]}$ also showed that higher BMI had a negative influence on IOL at term. Bishop score has proven to be a good predictor of successful induction in many studies. ${ }^{[12,13]}$ We noticed that women with a Bishop score $>6$ had a better success rate compared with women with a score $<5(p=0.272)$. Although Bishop score is an important predictor of the success of induction, the systematic review done by Kolkman et al. ${ }^{[14]}$ concluded that Bishop score, individually, was a poor predictor of the outcomes of induced labour. When the individual components of the Bishop score were analysed, it was observed that dilatation of the cervix was a better predictor $(p<0.001)$ compared with other factors of the Bishop score. ${ }^{[15]}$ Increased maternal age, shorter maternal height, greater BMI, greater weight gain during pregnancy, and initial cervical dilation $<3 \mathrm{~cm}$ are risk factors for CS after induction. ${ }^{[16]}$

The vaginal delivery and CS rates in the induced and spontaneous groups were similar to other studies. ${ }^{[3]}$ A study by Fisher et al. ${ }^{[17]}$ comparing the success rates of IOL over 3 phases in 20 years showed vaginal delivery rates of $79 \%, 72 \%$ and $71 \%$, respectively. The CS rates were higher in the nulliparous than in multiparous women. Systematic reviews have clearly shown that the CS rate is higher in the induced group than in the spontaneous group. However, a study by Grobman et al.$^{[8]}$ concluded that when induction is done at 39 weeks in low-risk women, the rate of CS was reduced compared with expectant management.

Failed induction $(46 \%)$, mostly seen in nulliparae was the most common indication for CS delivery. Nevertheless, it is not clear why certain cervices failed to dilate in primigravidae. Excluding failed induction, the common indications for CS delivery in both groups was fetal distress (35 v. 13). Fetal distress is comparatively higher in the induced group than the spontaneous group because pregnancies may have been interrupted for compromised fetuses owing to growth restriction, oligohydramnios, maternal hypertension or diabetes. Despite excluding the inductions for fetal compromise, the fetal distress rate was relatively higher $(10 \% ; n=13 / 128)$ compared with $4 \%(n=13 / 300)$ in spontaneously labouring women. Continuous electronic monitoring of the fetal heart in induced women is partially responsible for increased CS delivery for fetal distress.

Other factors that contributed to increased CS rates included a lack of a clear and objective definition of failed induction, ${ }^{[18]}$ patient's agony because of a prolonged process of induction, apprehension 
of the family and undue pressure on obstetricians to speed up the delivery also partially contributed to increased caesarean rates.

Surprisingly, there were more cases with meconium-stained liquor in the spontaneous group than in the induced group (12 v. 7), and similar observations were made by Macer et al. ${ }^{[19]}$

In third-stage complications, atonic postpartum haemorrhage was more prevalent in the induced group compared with the spontaneous group $(6.3 \%$ v. $4 \%)$. A study by Abisowa et al. ${ }^{[3]}$ reported similar findings $(4.5 \%$ v. $2.3 \%)$. Other complications that were comparable in both groups included retained placenta, perineal tear, puerperal fever and wound infections.

NICU admissions were more prevalent in the induced group than in the spontaneous group ( $4 \% \mathrm{v} .1 \%$ ). There were no neonatal deaths in either groups in the present study.

\section{Conclusion}

Multiparity, GA $>38$ weeks, BMI $<25 \mathrm{~kg} / \mathrm{m}^{2}$ and cervical dilatation are important predictors of successful vaginal delivery following induction.

It is a challenging task to strike a balance between reducing CS rates while ensuring the safety of mother/fetus from the assumed dangers of continuation of pregnancy. This can be accomplished by carefully selecting patients for induction, especially those with borderline indications and avoiding elective inductions. Factors that influence the success of induction can be used to counsel women with borderline and elective indications and their relatives in making decisions about mode of delivery as our study clearly showed an association between CS deliveries and the IOL, especially in nulliparous women.

All obstetric centres must periodically assess their CS rates, IOL indications, successful inductions and what factors affect these rates to reduce primary CS rates.

Declaration. None.

Acknowledgements. None.

Author contributions. RGB and PVB conceptualised the study. RGB, VV, NZ and SB acquired and analysed the data. All the authors wrote and revised the manuscript.

\section{Funding. None.}

Conflicts of interest. None.

1. Leduc D, Biringer A, Lee L, Dy J. Induction of Labor. J Obstet Gynaecol Canada 2013;35(9):840-857 https://doi.org/10.1016/S1701-2163(15)30842-2

2. Mishanina E, Rogozinska E, Thatthi T, Uddin-Khan R, Khan KS, Meads C. Use of labor induction and risk of cesarean delivery: A systematic review and meta-analysis. CMAJ 2014;186(9):665-673. https://doi.org/10.1503/cmaj.130925

3. Abisowo O, Oyinyechi A, Olusegun F, Oyedokun O, Motunrayo A, Abimbola O. Feto-maternal outcome of induced v. spontaneous labor in a Nigerian Tertiary Maternity Unit. Trop J Obstet Gynaecol 2017;34(1):21. https://doi.org/10.4103/TJOG.TJOG_59_16

4. National Institute for Health and Care Excellence (NICE). Inducing labor. London: NICE, 2008. https://www.nice.org.uk/guidance/cg70. (accessed 23 July 2015)

5. Vogel J, Souza J, Gülmezoglu A. Patterns and outcomes of induction of labor in Africa and Asia: A secondary analysis of the WHO Global Survey on Maternal and Neonatal Health. PLoS ONE 2013;8(6):e656129. https://doi.org/10.1371/journal.pone.0065612

6. Zenzmaier C, Leitner H, Brezinka C, Oberaigner W, König-Bachmann M. Maternal and neonatal outcomes after induction of labor: A population-based study. Arch Gynaecol Obstet 2017;295(5):11751183. https://doi.org/10.1007/s00404-017-4354-4

7. Bhat R, Kushtagi P. A re-look at the duration of human pregnancy. Singapore Med J 2006;47(12):10441048 .

8. Grobman WA, Rice MM, Reddy UM, et al. Labor induction v. expectant management in low-risk nulliparous women. N Engl J Med 2018;379(6):513-523. https://doi.org/10.1056/NEJMoal800566

9. Pevzner L, Rayburn WF, Rumney P, Wing DA. Factors predicting successful labor induction with dinoprostone and misoprostol vaginal inserts. Obstet Gynecol 2009;114(2):261-267. https://doi. org/10.1097/AOG.0b013e3181ad9377

10. Khan NB, Ahmed I, Malik A, Sheikh L. Factors associated with failed induction of labor in a secondary care hospital. J Pak Med Assoc 2012;62(1):6-10.

11. Dammer U, Bogner R, Weiss C, et al. Influence of body mass index on induction of labor: A historical cohort study. J Obstet Gynaecol Res 2018;44(4):697-707. https://doi.org/10.1111/jog.13561

12. Ikeotuonye AC, Anikwe CC, Obuna JA, et al. Relationship between Bishop score and success of induction of labor in federal teaching hospital, Abakaliki, Ebonyi State. Open J Obstet Gynaecol 2018;8(11):980-992. https://doi.org/10.4236/ojog.2018.811099

13. Teixeira C, Lunet N, Rodrigues $\mathrm{T}$, Barros $\mathrm{H}$. The Bishop score as a determinant of labor induction success: A systematic review and meta-analysis. Arch Gynaecol Obstet 2012;286(3):739-753. https:// doi.org/10.1007/s00404-012-2341-3

14. Kolkman DGE, Verhoeven CJM, Brinkhorst SJ, et al. The Bishop score as a predictor of labor induction success: A systematic review. Am J Perinatol 2013;30(8):625-630. https://doi org/10.1055/s-0032-1331024

15. Akyol A, Karademir O, Gedikbafl A, Cemal H, Ark AG. The role of the Bishop score for successful labor induction. Perinat J 2007;15(1):26-34.

16. Tolcher MC, Holbert MR, Weaver AL, et al. Predicting cesarean delivery after induction of labor among nulliparous women at term. Obstet Gynaecol 2015;126(5):1059-1068. https://doi.org/10.1097/ AOG.0000000000001083

17. Fischer R, Machac N, Hunter K, Adriance L. A comparison of induction of labor success rates over three time periods in 20 years at a single academic tertiary care center: Are we improving vaginal delivery rates? J Matern Neonatal Med 2018;31(7):907-913. https://doi.org/10.1080/14767058.201 7.1301422

18. Grobman WA, Bailit J, Lai Y, et al. Defining failed induction of labor. Am J Obstet Gynecol 2018;218:122.el-8. https://doi.org/10.1016/j.ajog.2017.11.556

19. Macer JA, Macer CL, Chan LS. Elective induction v. spontaneous labor: A retrospective study of complications and outcome. Am J Obstet Gynecol 1992;166:1690-1696. https://doi.org/10.1016/00029378(92)91558-r

Accepted 23 March 2021. 